\title{
DYNAMICS OF LINEAR OPERATORS CONNECTED WITH su(1,1) ALGEBRA
}

\section{V.E. KIM}

\begin{abstract}
In the present work we consider a linear continuous operator in a separable Fréchet space being one of the generators of Lie algebra $\mathrm{su}(1,1)$. We study the discretetime dynamical system generated by iterations of this operator. We show that under some additional conditions the operator that generates the indicated dynamical system is frequently hypercyclic and chaotic (in the sense of Devaney). Applications of this result to a study of specific operators are indicated.
\end{abstract}

Keywords: frequently hypercyclic operator, Lie algebra.

Mathematics Subject Classification: $47 \mathrm{~A} 16$

\section{INTRODUCTION}

Among dynamical systems with discrete time there is an important subclass comprising systems described by iterations of some mapping (see, for instance, [1], [2]). Let $X$ be a separable Fréchet space, $T: X \rightarrow X$ be a continuous operator. Then iterations $\left\{T^{n}, n=\right.$ $0,1, \cdots\}$ define a discrete dynamical system in space $X$. There exist various approaches for defining chaotic dynamical system (see, for instance, [3]). In the present paper we shall make use of the Devaney's definition of chaotic mapping, see [1, 2]. Operator $T$ is chaotic (in Devaney sense) if the following conditions hold:

1) operator $T$ is topologically transitive, i.e., there exists an element $x \in X$ such that its orbit $\operatorname{Orb}(T, x)=\left\{T^{n} x, n=0,1, \cdots\right\}$ is a dense subset in $X$;

2 ) the set of periodic points of operator $T$ is dense in $X$.

We recall that a point $x \in X$ is called periodic for operator $T$ if there exists $n \in \mathbb{N}$ such that $T^{n} x=x$.

A linear topologically transitive operator is usually called hypercyclic operator. It is wellknown that the only topologically transitive operators in finite-dimensional spaces are non-linear operators. However, in infinite-dimensional spaces there exist wide classes of hypercyclic and linear chaotic operators. For instance, the well-known Godefroy-Shapiro theorem [4] states, in particular, that all convolution operators (except the multiplication by a constant) in the space of all entire functions $H(\mathbb{C})$ are hypercyclic and chaotic. It was shown in work [5] that these operators are also frequently hypercyclic.

The notion of frequently hypercyclic operator was first introduced in work [6]. Let $A \subset \mathbb{N}$ be a set. We denote by $\underline{\operatorname{dens}}(A)$ the lower density of set $A$, i.e.

$$
\underline{\operatorname{dens}}(A)=\lim \inf _{N \rightarrow \infty} \frac{\#\{n \in A: n \leqslant N\}}{N} .
$$

According to the definition given in work [6] a linear continuous operator $T: X \rightarrow X$ is called frequently hypercyclic if there exists an element $x \in X$ such that for each non-empty open

V.E. Kim, Dynamics of Linear operators CONNECTED With su( 1,1$)$ Algebra.

(C) KIM V.E. 2014.

The work is supported by RFBR (grants 11-01-00572, 11-01-97019).

Submitted July 18, 2013. 
subset $U \subset X$ one has $\underline{\operatorname{den}}\left\{n \in \mathbb{N}: T^{n} x \in U\right\}>0$. It is easy to see that each frequently hypercyclic operators is hypercyclic. However, there are examples of hypercyclic operators not being frequently hypercyclic. More detailed information about these and other questions of the dynamics for these operators can be found, for instance, in book [7].

In the series of works (see, for instance, [8]-[11]) there was studied an issue on which operators in space $H(\mathbb{C})$ apart from convolution operators are also hypercyclic. For instance, it was proven in work [11] that a linear continuous non-injective operator $T: H(\mathbb{C}) \rightarrow H(\mathbb{C})$ is hypercyclic if $T$ satisfies commutation relation

$$
\left[T, \frac{\mathrm{d}}{\mathrm{d} z}\right]=I
$$

where $I$ is the identity mapping. It is known that commutation condition like (1) generates Lie algebra called usually Heisenberg-Weil algebra (see, for instance, [12]). The aim of the present work is to show that the hypercyclic condition (as well as frequently hypercyclic and chaotic condition) is obeyed for the operators satisfying commutation conditions generating another Lie algebra, namely, algebra $\mathrm{su}(1,1)$. As it is known (see, for instance, [12]), generators $K_{0}$, $K_{-}, K_{+}$of algebra $\mathrm{su}(1,1)$ satisfy the following commutation conditions

$$
\left[K_{0}, K_{+}\right]=K_{+} ;\left[K_{0}, K_{-}\right]=-K_{-} ;\left[K_{-}, K_{+}\right]=2 K_{0} .
$$

The paper is organized as follows. In Section 1 we prove the main result, Theorem 2. In section 2 we provide examples demonstrating application of Theorem 2. These examples show that by Theorem 2 we can describe some new classes of hypercyclic and chaotic operators, as well as that this theorem implies some known results of hypercyclic operators.

\section{MAIN RESULTS}

In this section we shall formulate and prove the main result on hypercyclic and chaotic character of operator $K_{-}$satisfying commutation conditions (2). In the proof of this result we shall make of the following theorem proven in work [6].

Theorem 1 (F. Bayart, S. Grivaux). Let $X$ be a separable Fréchet space, $T$ be a linear continuous operator in $X$. Suppose there exists a dense subspace $X_{0} \subset X$ and a mapping $S: X_{0} \rightarrow X_{0}$ such that

i: series $\sum_{n=0}^{\infty} T^{n} x$ converges absolutely $\forall x \in X_{0}$;

ii: series $\sum_{n=0}^{\infty} S^{n} x$ converges absolutely $\forall x \in X_{0}$;

iii: $T S x=x, \forall x \in X_{0}$.

Then operator $T$ is frequently hypercyclic and chaotic.

To prove the main theorem we shall employ the following auxiliary lemma.

Lemma 1. Let $X$ be a separable Fréchet space, $K_{0}, K_{-}, K_{+}$are linear continuous operators in $X$ satisfying commutation conditions (2). Then for each $n \in \mathbb{N}$

$$
K_{0} K_{+}^{n}=n K_{+}^{n}+K_{+}^{n} K_{0} .
$$

Proof. We prove by induction. It follows from relations (2) that $K_{0} K_{+}=K_{+}+K_{+} K_{0}$. Hence, identity (3) is established for $n=1$ and this is the induction base. We take arbitrary $m \in \mathbb{N}$, $m>1$. Let us prove identity (3) for $n=m$ assuming that (3) holds for $n=m-1$, i.e.,

$$
K_{0} K_{+}^{m-1}=(m-1) K_{+}^{m-1}+K_{+}^{m-1} K_{0} .
$$

We have

$$
\begin{aligned}
K_{0} K_{+}^{m} & =K_{0} K_{+}\left(K_{+}^{m-1}\right)=K_{+}^{m}+K_{+} K_{0} K_{+}^{m-1} \\
& =K_{+}^{m}+(m-1) K_{+}^{m}+K_{+}^{m} K_{0}=m K_{+}^{m}+K_{+}^{m} K_{0} .
\end{aligned}
$$


The next theorem is the main result of the paper.

Theorem 2. Let $X$ be a separable Fréchet space, $\left\{p_{k}\right\}_{k=1}^{\infty}$ be a family of semi-norms defining a topology in space $X$. Let $K_{0}, K_{-}, K_{+}$be linear continuous operators on $X$ obeying commutation conditions (2). Suppose that there exists an element $x \in X \backslash\{0\}$ such that

A: $x \in \operatorname{ker} K_{-}$;

B: system $\left\{K_{+}^{n} x, n=0,1, \cdots\right\}$ is complete in $X$;

C: for each $k \in \mathbb{N}$

$$
\lim _{m \rightarrow \infty}\left(\frac{p_{k}\left(K_{+}^{m} x\right)}{(m !)^{2}}\right)^{1 / m}<1
$$

D: $2 K_{0} x=x$.

Then operator $K_{-}$is frequently hypercyclic and chaotic.

Proof. Let us prove first that

$$
K_{-} K_{+}^{n} x=n^{2} K_{+}^{n-1} x
$$

for each $n \in \mathbb{N}$. We argue by induction. It follows from relations (2) and Conditions $\mathbf{A}$ and D that $K_{-} K_{+} x=x$. Thus, (4) holds true for $n=1$. The induction base is proven. We take an arbitrary $m \in \mathbb{N}, m \geq 1$. Assuming that (4) is satisfied for $n=m$, let us prove (4) for $n=m+1$, i.e., that $K_{-} K_{+}^{m+1} x=(m+1)^{2} K_{+}^{m} x$. Employing Conditions $\mathbf{A}$ and $\mathbf{D}$, relation (2) and Lemma, we obtain

$$
\begin{aligned}
K_{-} K_{+}^{m+1} x & =K_{-} K_{+}^{m+1} x+K_{+}\left(m^{2} K_{+}^{m-1} x-K_{-} K_{+}^{m} x\right)=K_{-} K_{+}^{m+1} x+m^{2} K_{+}^{m} x-K_{+} K_{-}\left(K_{+}^{m} x\right) \\
& =K_{-} K_{+}^{m+1} x+m^{2} K_{+}^{m} x+2 K_{0} K_{+}^{m} x-K_{-} K_{+}^{m+1} x \\
& =m^{2} K_{+}^{m} x+2 K_{0} K_{+}^{m} x=m^{2} K_{+}^{m} x+2 m K_{+}^{m} x+K_{+}^{m}\left(2 K_{0} x\right)=(m+1)^{2} K_{+}^{m} x .
\end{aligned}
$$

Thus, we have established that identity (4) is satisfied for each $n \in \mathbb{N}$. We observe that by Condition $\mathbf{B}$ the set $X_{0}=\operatorname{span}\left\{K_{+}^{n} x, n=0,1, \cdots\right\}$ is dense in $X$. We take operator $K_{-}$as operator $T$ in Theorem 1 and let us show that the hypothesis of Theorem 1 holds.

It follows from relation (4) and Condition $\mathbf{A}$ that for each $y \in X_{0}$ there exists a number $M \in \mathbb{N}$ such that $K_{-}^{m} y=0$ as $m \geq M$. Hence, operator $K_{-}$obeys Condition $\mathbf{i}$ of Theorem 1 . On set $X_{0}$ we define a mapping $S$ as

$$
S K_{+}^{n}=\frac{K_{+}^{n+1}}{(n+1)^{2}} .
$$

Then as one can easily see, $K_{-} S y=y$ for each $y \in X_{0}$. Hence, Condition iii of Theorem 1 is satisfied.

We note that

$$
S^{m} K_{+}^{n} x=\frac{(n !)^{2} K_{+}^{n+m} x}{((n+m) !)^{2}} .
$$

Then it follows from Condition $\mathbf{C}$ that series

$$
\sum_{m=0}^{\infty} p_{k}\left(S^{m} K_{+}^{n}\right), \quad n=0,1, \ldots
$$

converge for each $k \in \mathbb{N}$. It yields that series $\sum_{m=0}^{\infty} S^{m} y$ converges absolutely $\forall y \in X_{0}$. Therefore, Condition ii of Theorem 1 is satisfied as well. Thus, operator $K_{-}$is frequently hypercyclic and chaotic. 


\section{EXAMPLES}

In this section we provide examples demonstrating application of Theorem 2 to studying the dynamics of certain operators. We first prove a result concerning description of generators for algebra su(1,1). As a corolllary we shall obtain results on hypercyclic and chaotic character of certain particular operator acting acting the space of entire functions $H(\mathbb{C})$ with the topology of uniform convergence on compact sets. We note that an equivalent description of the topology of space $H(\mathbb{C})$ can be given by means of a countable system of semi-norms $p_{k}(f)=\max _{|z| \leqslant k}|f(z)|$, $k \in \mathbb{N}$.

Theorem 3. Let $X$ be a topological vector space, $T, A$ be linear continuous operators in $X$ satisfying commutation condition

$$
[T, A]=I
$$

where $I$ is the identity mapping on $X$. Then operators $K_{-}=T+A T^{2}, K_{+}=A, K_{0}=$ $(1 / 2) I+$ AT satisfy commutation conditions (2), i.e., they are generators of algebra $\mathrm{su}(1,1)$.

Proof. We note that it follows from relation (5) that $\left[T, A^{n}\right]=n A^{n-1},\left[T^{n}, A\right]=n T^{n-1}, n \in \mathbb{N}$. Employing this fact, we obtain

$$
\begin{aligned}
{\left[K_{-}, K_{+}\right] } & =\left[T+A T^{2}, A\right]=[T, A]+\left[A T^{2}, A\right]=I+A T^{2} A-A^{2} T^{2} \\
& =I+A\left[T^{2}, A\right]=I+2 A T=2 K_{0} .
\end{aligned}
$$

Let us check the validity of other relations. We have

$$
\begin{aligned}
& {\left[K_{0}, K_{+}\right]=[(1 / 2) I+A T, A]=[(1 / 2) I, A]+[A T, A]=A T A-A^{2} T=A[T, A]=A=K_{+} ;} \\
& {\left[K_{0}, K_{-}\right]=\left[(1 / 2) I+A T, T+A T^{2}\right]=\left[A T, T+A T^{2}\right]=[A T, T]+\left[A T, A T^{2}\right] .}
\end{aligned}
$$

We note that

$$
\begin{aligned}
& {[A T, T]=A T^{2}-T A T=A T^{2}-T(T A-I)=A T^{2}-T^{2} A+T=-2 T+T=-T ;} \\
& {\left[A T, A T^{2}\right]=A T A T^{2}-A T^{2} A T=A T\left(A T^{2}-T A T\right)=A T(-T)=-A T^{2} .}
\end{aligned}
$$

We finally get

$$
\left[K_{0}, K_{-}\right]=-T-A T^{2}=-K_{-} .
$$

Thus, commutation relations (2) hold true.

Corollary 1. The operator $\Phi$ acting in space $H(\mathbb{C})$ by the rule

$$
\Phi f(z)=f^{\prime}(z)+z f^{\prime \prime}(z)
$$

is frequently hypercyclic and chaotic.

Proof. We observe that $\left[\frac{\mathrm{d}}{\mathrm{d} z}, \mathbf{z}\right]=I$, where $\mathbf{z}$ stands for the operator of multiplication by the independent variable $z$ in space $H(\mathbb{C})$. Hence, by Theorem 6 operator $\Phi$ is a generator of algebra $\operatorname{su}(1,1)$. It is well-known that system $\left\{\mathbf{z}^{n}(1)=z^{n}, n=0,1, \cdots\right\}$ is complete $H(\mathbb{C})$. Moreover, it is obvious, $\Phi(1)=0,\left(I+2 \mathbf{z} \frac{\mathrm{d}}{\mathrm{d} z}\right)(1)=1$. Thus, Conditions $\mathbf{A}, \mathbf{B}$, and $\mathbf{D}$ of Theorem 2 are satisfied. We note that

$$
\lim _{m \rightarrow \infty}\left(\frac{p_{k}\left(z^{m}\right)}{(m !)^{2}}\right)^{1 / m}=\lim _{m \rightarrow \infty} \frac{k}{(m !)^{2 / m}}=0, \forall k \in \mathbb{N} .
$$

Thus, Condition $\mathbf{C}$ of Theorem 2 is satisfied and thus operator $\Phi$ is frequently hypercyclic and chaotic.

We note that the hypercyclic character of operator (6) follows from the results of work [9], since it is a particular case of Gel'fond-Leont'ev operator.

In the next statement we establish new classes of hypercyclic and chaotic operators in space $H(\mathbb{C})$. 
Corollary 2. Let $T$ be an operator in space $H(\mathbb{C})$ acting by the rule $T f(z)=P\left(\frac{\mathrm{d}}{\mathrm{d} z}\right) f(z)-$ $z f(z)$, where $P \in H(\mathbb{C})$ is some polynomial. Then operator $\Phi=T+\frac{\mathrm{d}}{\mathrm{d} z} T^{2}$ is frequently hypercyclic and chaotic operator in space $H(\mathbb{C})$.

Proof. We note that $\left[T, \frac{\mathrm{d}}{\mathrm{d} z}\right]=I$ (see [9]). Therefore, by Theorem 6 operator $\Phi$ is a generator of algebra $\mathrm{su}(1,1)$. It follows from general of linear differential equations in complex plane that there exists a function $F \in H(\mathbb{C})$ such that $F \in \operatorname{ker} T, F \not \equiv 0$. It was shown in [9] that the system $\left\{F^{(n)}, n=0,1, \cdots\right\}$ is complete $H(\mathbb{C})$. Moreover, it is obvious, $\Phi(F)=0$, $\left(I+2 \frac{\mathrm{d}}{\mathrm{d} z} T\right)(F)=F$. Thus, Conditions $\mathbf{A}, \mathbf{B}$, and $\mathbf{D}$ of Theorem 2 are satisfied. We also note that for each $k \in \mathbb{N}$

$$
\lim _{m \rightarrow \infty}\left(\frac{p_{k}\left(F^{(m)}\right)}{(m !)^{2}}\right)^{1 / m} \leqslant \lim _{m \rightarrow \infty}\left(\frac{m ! p_{2 k}(F)}{k^{m}(m !)^{2}}\right)^{1 / m}=\lim _{m \rightarrow \infty} \frac{1}{k(m !)^{1 / m}}=0 .
$$

Thus, Condition $\mathbf{C}$ of Theorem 2 holds true. Therefore, operator $\Phi$ is frequently hypercyclic and chaotic.

Let us provide an example of a particular operator in space $H(\mathbb{C})$ satisfying the hypothesis of Corollary 2. If as $T$ we take the operator $T=\frac{\mathrm{d}}{\mathrm{d} z}-\mathbf{z}$, then as $F$ we can take the function $F(z)=\exp \left(z^{2} / 2\right)$. Then operator $\Phi$ reads as $\Phi f(z)=f^{\prime \prime \prime}(z)-z f^{\prime \prime}(z)-f^{\prime}(z)-z f(z)$. In accordance with Corollary 2 , this operator is frequently hypercyclic and chaotic in space $H(\mathbb{C})$.

\section{BIBLIOGRAPHY}

1. R. Devaney. An introduction to chaotic dynamical systems. Addison-Wesley, New York (1989).

2. M.W. Hirsch, S. Smale, R. Devaney. Differential equations, dynamical systems, and an introduction to chaos. Elsevier, Amsterdam (2004).

3. A.Yu. Kolesov, N.Kh. Rozov. On the definition of 'chaos'. Uspekhi matem. nauk. 64:4, 125-172 (2009). [Russ. Math. Surv. 64:4, 701-744 (2009).]

4. G. Godefroy, J.H. Shapiro. Operators with dense, invariant, cyclic vector manifolds. J. Funct. Anal. 98:2, 229-269 (1991).

5. A. Bonilla, K.-G. Grosse-Erdmann. On a Theorem of Godefroy and Shapiro. Int. Equat. Op. Theor. 56:2, 151-162 (2006).

6. F. Bayart, S. Grivaux. Frequently hypercyclic operators. Trans. Amer. Math. Soc. 358:11, 5083-5117 (2006).

7. K.-G. Grosse-Erdmann, A. Peris Manguillot. Linear chaos. Springer, Heidelberg (2011).

8. H. Petersson Supercyclic and hypercyclic non-convolution operators. J. Oper. Theor. 55:1, 133-151 (2006).

9. V.É. Kim. Hypercyclicity and chaotic character of generalized convolution operators generated by Gel'fond-Leont'ev operators. Matem. zametki. 85:6, 849-856 (2009). [Math. Notes. 85:6, 807-813 (2009).]

10. J. Conejero, V. Muller. On the universality of multipliers on $H(\mathbb{C})$. J. Approx. Theory. 162:5, 1025-1032 (2010).

11. V.E. Kim. Commutation relations and hypercyclic operators. Arch. Math. 99:3, 247-253 (2012).

12. A.M. Perelomov. Generalized coherent states and some of their applications. Uspekhi fiz. nauk. 123:9, 23-55 (1977). [Soviet Physics Uspekhi. 20:9, 703-720 (1977).]

Vitalii Eduardovich Kim,

Institute of Mathematics CC USC RAS,

Chernyshevskii str., 112,

450008, Ufa, Russia

E-mail: kim@matem.anrb.ru 